\title{
FLUORESCENCE
}

\section{Isolated rings do big things}

the two
mechanisms
work
synergistically
and
cooperatively
of biological systems owes a lot to organic fluorophores. When in need of an organic visible emitter, many of us will choose a large $\pi$-conjugated molecule. Yet, organics devoid of contiguous $\pi$-systems can also emit in the visible. These species are often referred to as 'clusteroluminogens', a title that hints at the interactions between small chromophores that give rise to this unusual 'clusteroluminescence'. Ben Zhong Tang and his team set out to understand this by studying model compounds featuring phenyl rings spaced by short aliphatic groups surprisingly good visible emitters - which they describe in the Journal of the American Chemical Society.

Small $\pi$-systems are not expected to emit visible light because the energies associated with their electronic transitions are large, typically falling in the ultraviolet. However, $\pi$-systems can exhibit aggregation-induced emission (AIE), a phenomenon traditionally explained in terms of restricted intramolecular motion in closely packed molecules. The imparted rigidity serves to supress non-radiative decay, thereby facilitating emission in high quantum yields. This 'classical' interpretation is not

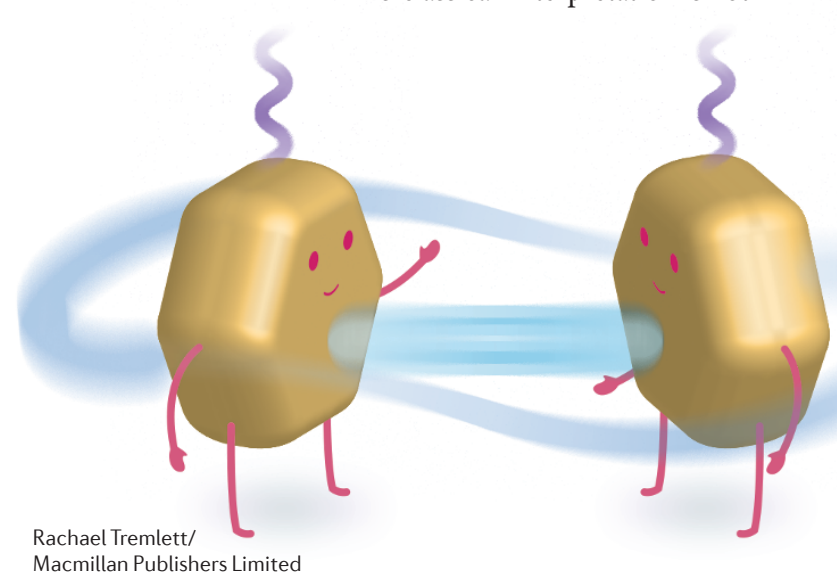

Macmillan Publishers Limited the only cause of AIE, as Tang and co-workers show. Although most consider $\pi$-systems separated by saturated carbon centres to be electronically localized, in certain geometries, the $\pi$-systems can be conjugated through space. For example, two $\mathrm{Ph}$ rings that are not $\pi$-conjugated can nevertheless interact electronically when they are near each other and are somewhat parallel. In this case, AIE is a result of both increased rigidity and throughspace conjugation, and Tang says that "the two mechanisms work synergistically and cooperatively".

When in solution, the aryls in bis(2,4,5-trimethylphenyl)methane (DPM) undergo facile rotation, interrupting through-space $\pi-\pi$ interactions, such that photo-excited DPM decays primarily through a nonradiative pathway. Indeed, DPM in THF exhibits an absorption at $277 \mathrm{~nm}$, with a weak emission at $297 \mathrm{~nm}$ being characteristic of isolated phenyl rings. Making use of the hydrophobic effect, Tang's team added $\mathrm{H}_{2} \mathrm{O}$ to the mix, causing DPM to aggregate and emit more strongly both at $297 \mathrm{~nm}$ and, importantly, in the visible region at $\sim 380 \mathrm{~nm}$. In 9:1 $(v / v) \mathrm{H}_{2} \mathrm{O}-\mathrm{THF}$, the latter band is two orders of magnitude stronger than it is for DPM in pure THF. Long-wavelength AIE is also observed in a solid DPM derivative, which emits at $470 \mathrm{~nm}$ in up to $70 \%$ quantum yield. Absorption spectra of clusteroluminogens like DPM and 1,1,2,2-tetraphenylethane are unaffected by through-space interactions, and Tang notes that "simulations on these molecules confirm that through-space conjugation is present in the excited but not the ground state". Indeed, the HOMO of 1,1,2,2-tetraphenylethane is localized on two geminal $\mathrm{Ph}$ rings, while the LUMO also extends over the space between the rings.

Through-space conjugation enables us to rationalize, for example, how poly((maleic anhydride)-alt(vinyl acetate)) exhibits strong emission and solvatochroism. Although not $\pi$-conjugated, the polymer exhibits electronic delocalization of otherwise isolated $\pi$-systems through $n-\pi^{\star}$ interactions between anhydride and acetate $\mathrm{O}$ atoms. Similarly, two adjacent polypeptides can be conjugated through $n-\pi^{*}$ interactions between $\mathrm{N}$ atoms and $\mathrm{C}=\mathrm{O}$ groups, as well as through hydrogen bonding. These materials are not formally $\pi$-conjugated, but through-space conjugation sees them fluoresce at longer wavelengths than one might predict.

Tang and his team are now investigating clusteroluminogens featuring heteroaryls with different spacers, as well as biogenic (macro) molecules free of aryl rings, to expand our knowledge of through-space conjugation and its effects on luminescence. The molecules studied by Tang may represent more soluble and biocompatible luminogens than the large planar polyaromatics commonly used for imaging. Because throughspace conjugation is sensitive to the (intermolecular or intramolecular) distance between moieties, it becomes possible to perturb conjugation through external stimuli such as mechanical force. Tang predicts that "we can build a relationship between the magnitude of mechanical force and the luminescence intensity, from which smart mechanically responsive materials can be developed".

David Schilter 